\title{
CFD analysis of the two-phase bubbly flow characteristics in helically coiled rectangular and circular tube heat exchangers
}

\author{
Alamin Hussain ${ }^{1}$, Andrew M. Fsadni ${ }^{2, a}$ \\ ${ }^{1,2}$ School of Engineering, Kirkham Room 124, University of Central Lancashire, Preston, PR1 2HE, UK
}

\begin{abstract}
Due to their ease of manufacture, high heat transfer efficiency and compact design, helically coiled heat exchangers are increasingly being adopted in a number of industries. The higher heat transfer efficiency over straight pipes is due to the secondary flow that develops as a result of the centrifugal force. In spite of the widespread use of helically coiled heat exchangers, and the presence of bubbly twophase flow in a number of systems, very few studies have investigated the resultant flow characteristics. This paper will therefore present the results of CFD simulations for the two-phase bubbly flow in helically coiled heat exchangers as a function of the volumetric void fraction and the tube cross-section design. The CFD results are compared to the scarce flow visualisation experimental results available in the open literature.
\end{abstract}

\section{Introduction}

Helically coiled tube heat exchangers have seen extensive use in industrial applications such as in refrigeration, condensers and evaporators in the food industry, heat recovery systems, power generation, process plants and the nuclear industry where helically coiled heat exchangers are used for residual heat removal systems. This is due to the fact that helically coiled heat exchangers have a greater heat transfer rate as well as a more compact design compared to straight tube heat exchangers $[1,2]$.

Micro bubble formation is a phenomenon caused by water supersaturated with dissolved air, consequently, leading to bubble nucleation on the walls of the heat exchanger. Bubble nucleation occurs at low temperatures such as when the air can absorb the largest magnitude of gas $[3,4]$. For example, in a domestic boiler system, bubble nucleation will occur when the boiler is switched on in the morning. Micro bubble formation affects many of the aforementioned industries. Computational investigations on simulating the effect of micro bubble formation in heat exchangers can lead to design improvements of deaerators which will, as a result, improve the efficiency of the heat exchangers [5]. Bubbles accumulating within the heat exchanger can result in cold spots, reducing the area in which heat transfer can occur thus reducing the efficiency of the heat exchanger and the overall heat transfer coefficient. Furthermore, a comprehensive knowledge of the behaviour of the bubbles such as the second phase distribution within the heat exchangers, is essential for an accurate representation of the heat exchanger's performance.

A bubbly flow in a heat exchanger is the result of the micro bubbles detaching from the walls of the heat exchanger and into the flow system. Two-phase bubbly flow is characterised by the presence of the bubbles with a maximum size much smaller than that of the containing vessel or duct. Furthermore the bubbles are dispersed in a continuous water phase [6].

Previous studies in this field, are mostly theoretical. However there have been some experimental investigations carried out. The lack of experimental data can be due to difficulties in obtaining reliable data on bubbly flow and bubble nucleation, as well as the inherent complexity associated with two-phase flows making the data difficult to analyse [5, 7].

In this paper we will investigate the phase distribution of bubbly flow in a helically coiled tube heat exchanger using computational fluid dynamics (CFD) at system conditions typical of domestic wet central heating systems. Due to the difficulties related to experimental methods and the cost and investment needed, the development and validation of a CFD simulation using existing experimental data, may prove beneficial for the development of helically coiled tube heat exchangers.

\section{CFD Modelling}

\footnotetext{
${ }^{\mathrm{a}}$ Corresponding author: afsadni@uclan.ac.uk
} 
The simulation was modelled using the ANSYS version 15 CFD software package. The mesh was developed using the ANSYS design modeller's meshing tools and the fluid flow was modelled using the FLUENT solver. The 3D model of the two helical pipes, one with a circular cross-section and one with a rectangular crosssection, were imported into ANSYS as a STEP file which was initially modelled in Pro/E wildfire. Three meshes of each 3D-model were produced with element counts of circa $2 \mathrm{e}+6,3.5 \mathrm{e}+6$ and $5 \mathrm{e}+6$ elements. The initial simulations of the rectangular pipes took between 1000 and 1300 iterations to converge, and the initial simulations of the circular pipes took between 600 and 1000 iterations to converge. The "initial simulations" refer to the first group of multi-phase flow simulations carried out on the pipes, the setup of which is specified below. The Reynolds number of the circular pipe was 2500 and the Reynolds number of the rectangular pipe was 3225 , suggesting both flows through the pipes are transient flows. For both cases, the calculations were done under the assumption that the presence of air has a negligible effect on the overall fluid properties. Figures 1 $\& 2$ illustrate the 3D models of the tubes used in the present study.

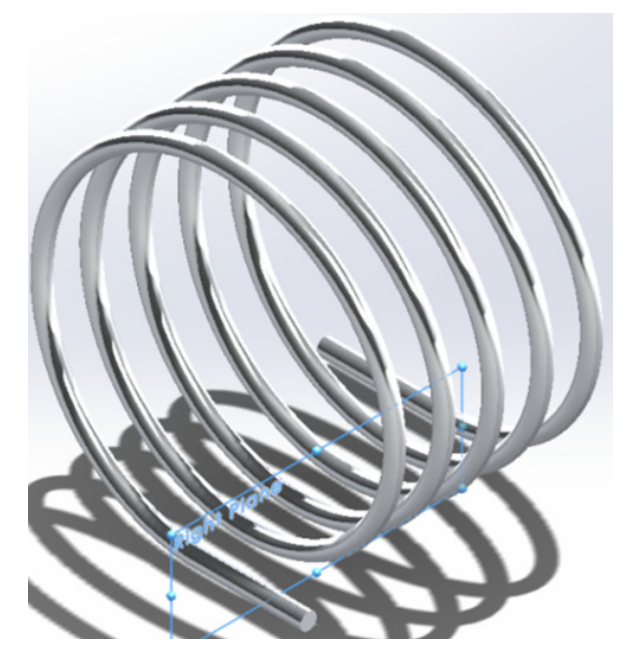

Figure 1. 3D model of helically coiled tube with circular crosssection

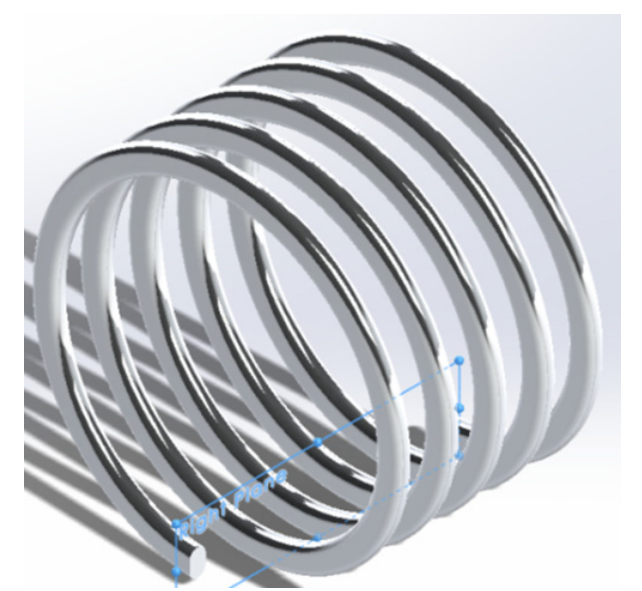

Figure 2. 3D model of helically coiled tube with rectangular cross-section

\subsection{Model setup - FLUENT}

The two-phase flow was simulated using one of the multiphase methods available in FLUENT, namely the mixture method. It was reported that for bubbly flows the mixture method was the most suitable, hence the use of this method in the study [8].

The solver type was a pressure based solver, which enabled the pressure based Navier Stokes solutions. The flow was assumed to be in a steady state, implying that the solution is independent to time, and a gravitational force was applied in the relevant direction in order to include the buoyancy of the bubbles in the simulation.

The turbulence model used was the realizable $k-\varepsilon$ turbulence model with standard wall functions used as the near wall treatment. The model also assumes isotropic turbulence. The realizable $k-\varepsilon$ turbulence model is a significant improvement to the standard $k-\varepsilon$ turbulence model in almost all cases, thus suggesting that the realizable model will produce more accurate results [9]. The partial differential equation is closed using the realizable $k-\varepsilon$ turbulence model where one equation is solved for the turbulence kinetic energy $k$ as in equation (1) and another equation is solved for the turbulent dissipation $\varepsilon$ as in equation (2).

$$
\begin{aligned}
& \frac{\partial}{\partial t}(\rho k)+\frac{\partial}{\partial x_{j}}\left(p k u_{j}\right)=\frac{\partial}{\partial x_{j}}\left[\left(\mu+\frac{\mu_{t}}{\sigma_{k}}\right) \frac{\partial k}{\partial x_{j}}\right]+ \\
& P_{k}+P_{b}-\rho \varepsilon-Y_{M}+S_{k} \\
& \frac{\partial}{\partial t}(\rho \varepsilon)+\frac{\partial}{\partial x_{j}}\left(p \varepsilon u_{j}\right)=\frac{\partial}{\partial x_{j}}\left[\left(\mu+\frac{\mu_{t}}{\sigma_{\varepsilon}}\right) \frac{\partial \varepsilon}{\partial x_{j}}\right]+ \\
& \rho C_{1} S_{\varepsilon}-\rho C_{2} \frac{\varepsilon^{2}}{k+\sqrt{v \varepsilon}}+C_{1 \varepsilon} \frac{\varepsilon}{k} C_{3 \varepsilon} P_{b}+S_{\varepsilon}
\end{aligned}
$$

where $C_{1}$ is calculated using equation (3), $\eta$ is given by equation (4) and $S$ is the deformation tensor found using equation (5).

$$
\begin{aligned}
& C_{1}=\max \left[0.43, \frac{\eta}{\eta+5}\right], \\
& \eta=S \frac{k}{\varepsilon} \\
& S=\sqrt{2 S_{i j} S_{i j}} .
\end{aligned}
$$

In these equations $P_{b}$ is the generation of the turbulence kinetic energy calculated using equation (7), and $P_{k}$ is the generation turbulence kinetic energy due to the mean velocity gradients calculated using equation (6).

$$
\begin{aligned}
& P_{k}=\mu_{t} S^{2} \\
& P_{b}=\beta g_{i} \frac{\mu_{t}}{P r_{t}} \frac{\partial T}{\partial x_{i}}
\end{aligned}
$$

where $P r_{t}$ is the Prandtl number of a turbulent flow, which for the realizable model is $0.85, \mathbf{g}_{\mathbf{i}}$ is the vector of the gravitational component in the $I^{\text {th }}$ direction, and $\beta$ is 
the thermal expansion coefficient, which is defined by equation (8).

$$
\beta=-\frac{1}{\rho}\left(\frac{\partial \rho}{\partial T}\right)_{p}
$$

The turbulent viscosity $\mu_{t}$ is found using equation (9).

$$
\mu_{t}=\rho C_{\mu} \frac{k^{2}}{\varepsilon}
$$

where $C_{\mu}$ is a function of the mean strain and rotation rates, the angular velocity of the system rotation and the turbulence fields modelled using the following equations,

$$
\begin{aligned}
C_{\mu} & =\frac{1}{A_{0}+A_{s} \frac{k U^{*}}{\varepsilon}}, \\
U^{*} & =\sqrt{S_{i j} S_{i j}+\widetilde{\Omega}_{i j} \widetilde{\Omega}_{i j}}, \\
\widetilde{\Omega}_{i j} & =\Omega_{i j}-2 \varepsilon_{i j k} \omega_{k}, \\
\Omega_{i j} & =\overline{\Omega_{l j}}-\varepsilon_{i j k} \omega_{k},
\end{aligned}
$$

where $\overline{\Omega_{\iota \jmath}}$ is, in a rotating reference frame, the mean rate of rotation tensor viewed, with angular velocity $\omega_{k}$. The model constants have the following values;

$C_{1 \varepsilon}=1.44, \quad C_{2}=1.9, \quad \sigma_{k}=1.0, \quad \sigma_{\varepsilon}=1.2$, $A_{0}=4.04, \quad A_{s}=\sqrt{6} \cos \phi$

In this case $A_{s}$ is found using the following equations;

$$
\begin{aligned}
& \phi=\frac{1}{3} \cos ^{-1}(\sqrt{6} W), \\
& W=\frac{S_{i j} S_{j k} S_{k i}}{\widetilde{S}^{3}}, \\
& \tilde{S}=\sqrt{S_{i j} S_{i j}}, \\
& S_{i j}=\frac{1}{2}\left(\frac{\partial u_{j}}{\partial x_{i}}+\frac{\partial u_{i}}{\partial x_{j}}\right) .
\end{aligned}
$$

The primary phase was water with the secondary phase being air. The air bubble diameter was specified as $0.2 \mathrm{~mm}$ and the bubbles were assumed to be spherical in shape. The drag law selected was the Schiller-Neumann as this was the default method and is acceptable for general fluid to fluid multiphase simulations [11].

As both the air and water were specified to have the same initial velocity both were also assumed to have the same change in velocity as the fluids travelled through the pipe. If both fluids are assumed to have the same change in velocity a further assumption that can be made, is that there is no slip between the water and air. Therefore when specifying the slip model of the phase interaction the selection was changed from the standard option (manninen et al.) to none.
The velocity of both the water and the air at the inlet was $0.25 \mathrm{~m} \mathrm{~s}^{-1}$ and the volume void fraction was $1.05 \mathrm{e}-4$. The pressure of the system was $270 \mathrm{kPa}$, the turbulent intensity being $5 \%$ and hydraulic diameter $12.9 \mathrm{~mm}$. The SIMPLE (Semi-Implicit Method for Pressure Linked Equations) scheme was used for the pressure-velocity coupling as this was the standard coupling method and for complicated flows such as turbulent flows the SIMPLE and SIMPLEC (SIMPLE-Consistent) schemes will converge at similar rates [12].

The first order discretization scheme used for the momentum, volume fraction, turbulent kinetic energy and turbulent dissipation rate, while the PRESTO and least squares cell based schemes were used for the pressure and the gradient respectively. The residual convergence was set at $1 \mathrm{e}-5$. The solution was initialised using the hybrid initialisation method.

Table 1. Simulation Parameters for both groups of Simulations

\begin{tabular}{|c|c|c|}
\hline Parameters & Initial simulation & VF 0.05 \\
\hline $\begin{array}{c}\text { V(both phases }- \text { no } \\
\text { slip) }\left(\text { m }^{-1}\right)\end{array}$ & 0.25 & 0.25 \\
\hline $\begin{array}{c}\text { Volumetric void } \\
\text { fraction of air }\end{array}$ & $1.05 \mathrm{e}-4$ & 0.05 \\
\hline $\boldsymbol{D}_{\boldsymbol{b}}($ mm $)$ & 0.2 & 0.2 \\
\hline $\boldsymbol{P}(\boldsymbol{k P a})(\boldsymbol{a b s})$ & 270 & 270 \\
\hline Multiphase model & Mixture & Mixture \\
\hline $\begin{array}{c}\text { Convergence residual } \\
\text { value }\end{array}$ & $1 \mathrm{e}-5$ & $1 \mathrm{e}-5$ \\
\hline $\begin{array}{c}\text { Simulation order } \\
\text { scheme }\end{array}$ & First order & Second order \\
\hline
\end{tabular}

As can be seen from table 1 two sets of simulation parameters were modelled. The first being detailed above and the second seeing two changes. The first change was to increase the volumetric void fraction of the air, the second being changing the simulation method scheme from first order to, second order for the momentum, turbulent kinetic energy and turbulent dissipation rate, and the QUICK scheme was used for the volume fraction. The purpose for changing the scheme is that the second order and QUICK schemes should produce more accurate results from the simulations.

A further simulation was performed with a velocity increase from $0.25 \mathrm{~m} \mathrm{~s}^{-1}$ to $1 \mathrm{~m} \mathrm{~s}^{-1}$ changing the flow from transient to turbulent. As a result the number of iterations for the simulation to converge increased to a point where the simulation was taking too long to complete and thus was prematurely ended.

\section{Results and Discussion}

The results of the CFD simulations as detailed above are shown in figures $3-6$. The figures all show the volume fraction of the air within the flow. The labels shown on figure 3 show, at what point of the pipe the cross-sections contours appear at. The inlet and outlet are at the point where the fluids flow into the pipe and out of the pipe respectively. Loop $1-4$ refers to how many times the fluids have travelled a full loop within the pipe, returning to a point that is parallel to the inlet point. The results of 
the other simulations will be shown similarly to what is shown in figure 3 .

Figure 3 illustrates the volume fraction distribution of the air within the circular helical pipe with mesh elements counts of $5 \mathrm{e}+6$ elements. The simulation parameters were that of the initial simulation shown in Table 1 . The figures display cross sections of the pipe from the inlet to the outlet at every point the pipe loops. Figure 3 shows that the distribution of the volume fraction is rather evenly distributed across the cross section of the pipe, showing no tendency for the air to separate from the water or for the air bubbles to accumulate at any point within the pipe. Furthermore, the void fraction distribution suggests that the centrifugal force affects the air and water to a similar extent.
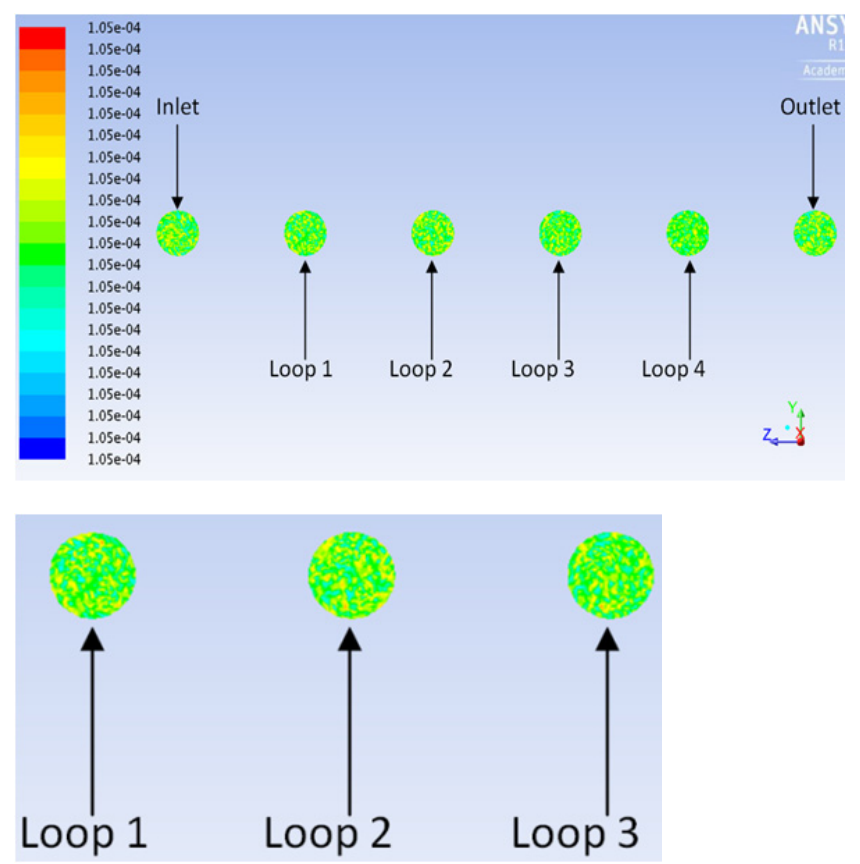

Figure 3. Volume void fraction distribution of air within the circular pipe under initial simulation conditions with enlarged sections for loops $1-3$.

Figure 4 illustrates the same series of simulations as in figure 3 but the pipe model is different. Where figure 3 uses the circular cross-sectioned pipe, figures 4 uses the rectangular cross-sectioned pipe, and in both cases the element count is $5 \mathrm{e}+6$ elements.
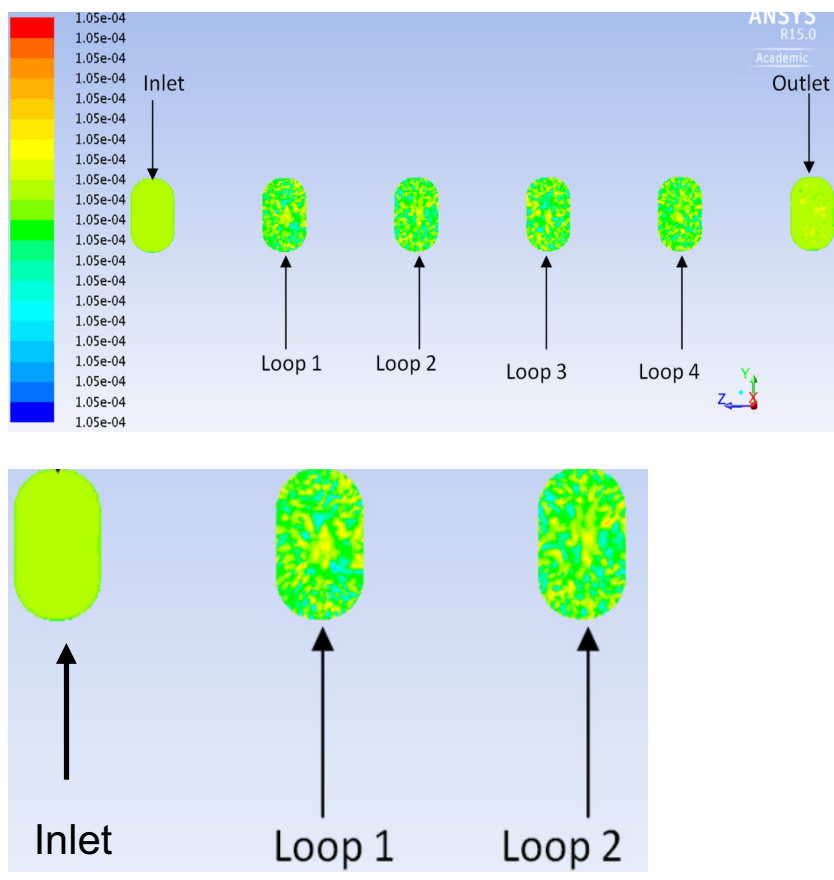

Figure 4. Volume void fraction distribution of air within the rectangular pipe under initial simulation conditions with enlarged sections for the inlet and loops $1 \& 2$.

The results shown in each series of simulations are very similar, in that the volume fraction distribution is spread throughout the pipe thus suggesting that the air and water phases are not separating.

A difference that is noticeable between the rectangular pipes results and the circular pipes results is that, the inlet and outlets on the rectangular pipes show a far more uniform distribution. This is likely because the plane at which the contours are applied to are much closer to the model's actual inlet and outlet points for the rectangular pipes, whereas the plane for the circular pipes lies a few millimetres into the pipe from the inlet and outlet. This is due to the fact that circular pipe has a short lead in before the pipe starts to coil and the rectangular pipe doesn't. The following figures illustrate the results from the simulations carried out with a volumetric void fraction of 0.05 and the solution methods being second order as well as having an element count of 5 e+6 elements for both figures. 

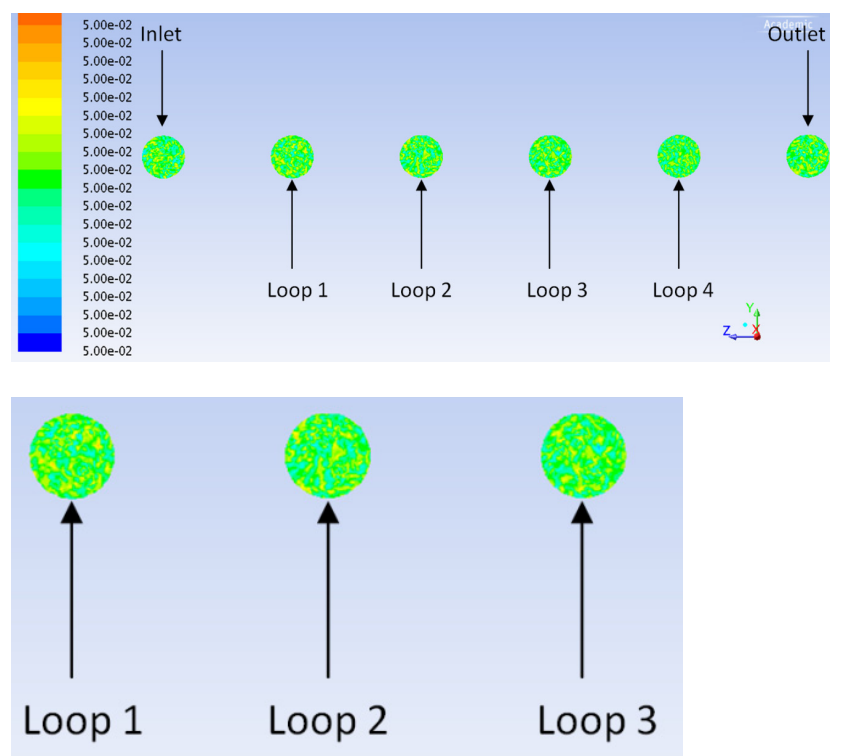

Figure 5. Volume void fraction distribution of air within the circular pipe with a volume fraction of 0.05 with enlarged sections for loops $1-3$.

Figure 5 illustrates the results for the circular crosssection tube, where almost no change in the volume fraction distribution than what is seen in figure 3. This suggests that the increase in the volume fraction and the change of the solution method to a second order type have resulted in no significant change in the volume fraction distribution. Figure 6 illustrates the volume fraction distribution for the rectangular cross-sectioned pipe. This shows a very similar volume fraction distribution as in figure 4, further reinforcing the idea that changing the volume fraction magnitude has little effect on the volume fraction distribution. It was expected that the centrifugal force would cause the bubbles to shift towards the inner part of the pipe/centre of the coil. In figures $2-6$ there would be a higher volume fraction to the top of the contoured cross-sections. However the volume fraction distribution seen in figures $2-6$ may be due to the Reynolds number. As a recent experimental two-phase investigation reported, with increasing Reynolds number, the bubble distribution within the fluid becomes more homogenous [13]. As mentioned before the Reynolds number of the flows lied within the transitional state, the Reynolds numbers being calculated without taking the air bubbles into account. The presence of air would likely increase the Reynolds number as the dynamic viscosity of air is smaller than that of water [14].

Another factor that could affect whether the flow is turbulent or not, is torsion. It was found that torsion can destabilize the flow and reduce the Reynolds number at which turbulent flows occur, which could result in a flow that is transitional in a straight pipe, being turbulent in helically coiled pipe [1]. The scale for the contours shown on figures $2-6$ do not change. This is because the scale is shown to three significant figures. This suggests that there is a less than $1 \%$ change in volume fraction, further reinforcing the idea that the volume fraction distribution is homogenous.

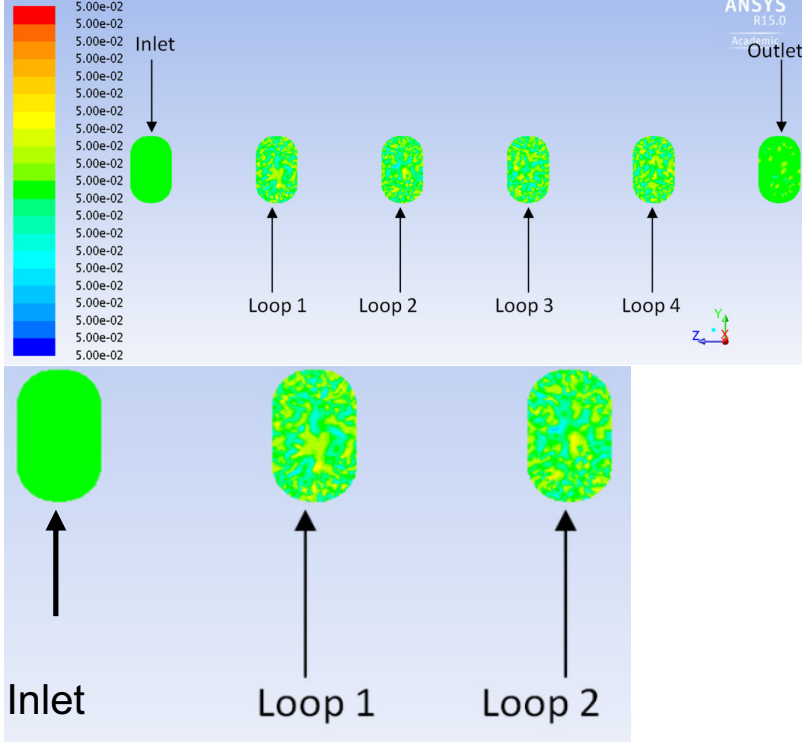

Figure 6. Volume void fraction distribution of air within the rectangular pipe with a volume fraction of 0.05 with enlarged sections for loops $1-3$.

The reason figures $5 \& 6$ show similar volume fraction distributions to figures $3 \& 4$ is because changing the bulk fluids volumetric void fraction has a minimal effect on the actual volume fraction distribution in the tube [5]. A possible way of improving the CFD simulation and the results found could involve the application of a different two-phase modelling scheme, namely the Eulerian model. When using the mixture model, pseudo properties are used to solve a single set of conservation equations. But when using the Eulerian model each of the phases are solved using a complete set of conservation equations. Furthermore for a well-mixed gas-liquid region, it has been reported that using the mixture model will not yield correct results, due to the centrifugal and Coriolis forces. Therefore the Eulerian model should be used for future modelling [1].

Further improvements to the CFD simulations carried out would be to run simulations with varying Reynolds number from circa 100 to 10,000 to further validate the simulations using the idea that the phase distribution becomes more homogenous with increasing Reynolds number [13]. This would be carried out by changing either the velocity of the flow or the hydraulic diameter of the pipes.

\section{Conclusions}

This paper has presented a CFD simulation study on the volume fraction distribution of a two-phase bubbly flow for two helically coiled heat exchangers, one with a circular cross-section and the other having a rectangular cross-section under system conditions typical of a domestic wet central heating system. The present study has suggested that the volume fraction distribution found through the CFD simulation does resemble that found in experimental results assuming that there are factors that have affected the Reynolds number that were not taken into account when initially calculating the Reynolds 
number. This is further shown by the fact that the volume fraction distribution does not change when the air volume void fraction value is changed.

The importance of this study lies with the fact that having a thorough understanding of the volume fraction distribution for bubbly flows within heat exchangers should lead to enhanced deaeration system efficiencies and hence less energy would be needed for the heat exchangers to perform as desired. Furthermore having a validated CFD simulation for bubbly flow within heat exchangers will help develop heat exchangers as experimental methods are difficult, costly and time consuming. Finally, due to the widespread use of helically coiled heat exchangers having a validated CFD simulation model of such heat exchangers will greatly aid the numerous industries that utilise and depend on helically coiled heat exchangers. Further studies that could be performed to build upon what has been found in this paper include the application of a different multiphase simulation model such as the Eulerian model to investigate how the Eulerian model and the mixture model differ as well as changing the velocity to alter the system Reynolds number.

\section{Notation List}

$C_{\mu} \quad$ Function of the mean strain and rotation rates, the angular velocity of the system rotation and the turbulence fields (-)

$D \quad$ Pipe Diameter (m)

$D_{b} \quad$ Bubble Diameter (m)

$G_{b} \quad$ Generation of turbulent kinetic energy due to the buoyancy of the second phase $\left(\mathrm{J} / \mathrm{m}^{3} \mathrm{~s}\right)$

$G_{k} \quad$ Generation of the turbulent kinetic energy due to the mean velocity tensor $\left(\mathrm{J} / \mathrm{m}^{3} \mathrm{~s}\right)$

$k \quad$ Turbulent kinetic energy $\left(\mathrm{m}^{2} / \mathrm{s}^{2}\right)$

$P \quad$ System pressure $(\mathrm{Pa})$

$P_{g} \quad$ Partial pressure of dissolved gas $(\mathrm{Pa})$

$S \quad$ Deformation tensor (-)

$T \quad$ Fluid temperature $\left({ }^{\circ} \mathrm{C}\right)$

$V \quad$ Fluid Velocity $\left(\mathrm{m} \mathrm{s}^{-1}\right)$

$V F \quad$ Volumetric void fraction (-)

$g_{i} \quad$ Vector of the gravitational component (-)

$\mathrm{Pr}_{t} \quad$ Prandtl number (-)

$P_{b} \quad$ The generation of the turbulence kinetic energy (J / $\left.\mathrm{m}^{3} \mathrm{~s}\right)$

\section{Greek letters}

$\alpha \quad$ Saturation ratio, (-)

$\beta \quad$ Thermal expansion coefficient $\left(\mathrm{K}^{-1}\right)$

$\gamma$ Surface tension, $(\mathrm{N} / \mathrm{m})$

$v \quad$ Kinematic viscosity, $\left(\mathrm{m}^{2} / \mathrm{s}\right)$

$\rho \quad$ Density of fluid $\left(\mathrm{kg} / \mathrm{m}^{3}\right)$

$\varepsilon \quad$ Turbulent dissipation $\left(\mathrm{m}^{2} / \mathrm{s}^{3}\right)$

$\sigma_{k} \quad$ Turbulent Prandtl numbers for $k(-)$

$\mu_{t} \quad$ The turbulent viscosity $(\mathrm{kg} / \mathrm{m} \mathrm{s})$

$\sigma_{\varepsilon} \quad$ Turbulent Prandtl numbers for $\varepsilon(-)$

$\overline{\Omega_{\imath \jmath}} \quad$ Rotating reference frame

$\omega_{k} \quad$ Angular velocity $\left(\mathrm{rad} \mathrm{s}^{-1}\right)$

\section{Acknowledgments}

The authors would like to thank the University of Central Lancashire, for supporting this research work.

\section{References}

1. J.S. Jayakumar, Helically Coiled Heat Exchangers, Heat Exchangers - Basics Design Applications, Dr. Jovan Mitrovic (Ed.), ISBN: 978-953-51-0278-6, InTech, (2012), Available from:

http://www.intechopen.com/books/heat-exchangersbasics-design-applications/helically-coiledheatexchangers. Last accessed (12/08/2015)

2. R. Manna, J.S. Jayakumar, R.B. Grover, Thermal Hydraulic design of a condenser for a natural circulation system, J. energy, heat and mass transfer, 18, 39-46 (1996)

3. Gerrard W, (1976). Solubility of gases and liquids. New York: Plenum Press.

4. C.L. Young, R. Battino, H.L. Clever, The solubility of gases and liquids - introductory information, Nitrogen and Air, solubility data series 10, Oxford: Pergamon Press (1982).

5. A.M. Fsadni, Y.T. Ge, Experimental and CFD analysis of two phase distribution in horizontal pipes for enhanced system deaeration. In: International Conference on Applied Energy, 5-8 ${ }^{\text {th }}$ July 2012, Suzhou, China (2012)

6. R.H.S. Winterton, J.S. Munaweera, Bubble size in two-phase gas-liquid bubbly flow in ducts,

Chemical engineering and processing 40, p437-447 (2012).

7. N.J., Hepworth, J.W.R. Boyd, J.R.M. Hammond, J. Varley, Modelling the effect of liquid notion on bubble nucleation during beer dispense. Chemical engineering sciences. 58, 4071-4084 (2006)

8. N. Basu, A. Troshko, G. Nurnberg, Modelling of two-phase flow and boiling with FLUENT (2006) Available from: http://www.scribd.com/doc/3754733/Basu . Last accessed 05/08/2015.

9. T.H. Shih, W.W. Liou, A. Shabbir, Z. Yang, J. Zhu, A new k- $\varepsilon$ eddy viscosity model for high Reynolds number turbulent flows - model development and validation, Computers and Fluids. 24 (3), 227-238 (1995)

10. CFD-online, Realisable k-epsilon model. Available from: http://www.cfd-

online.com/Wiki/Realisable_k-epsilon_model . Last accessed 25/08/2015 (2015)

11. ANSYS (2009). Setting Up the Eulerian Model, ANSYS FLUENT 12.0 User's Guide. United States, (2009)

12. Sharcnet, Choosing the Pressure-Velocity Coupling Method. Available from:

https://www.sharcnet.ca/Software/Fluent6/html/ug/n ode1021.htm. Last accessed 25/08/2015 (2006)

13. H. Saffari, R. Moosavi, E. Gholami, N.M. Nouri, The effect of bubble on pressure drop reduction in 
EFM 2015

helical coil. Experimental Thermal and Fluid

Science. 1 (51), p251-256 (2013)

14. Engineeringtoolbox, Dynamic, Absolute and

Kinematic Viscosity. Available from:

http://www.engineeringtoolbox.com/dynamicabsolute-kinematic-viscosity-d_412.html. Last accessed 04/09/2015 (2015) 